\title{
The Vertex Graphical Condensation for Algebraic Structure Count of Molecular Graphs
}

\author{
Luzhen Ye \\ School of Sciences, Jimei University, Xiamen 361021, China \\ lzye555@sina.com.
}

(Received November 19, 2021)

\begin{abstract}
The algebraic structure count of a bipartite graph $G=(U, V)$, denoted by $L(G)$, is defined as the difference between the number of so-called "even" and "odd" Kekulé structures of $G$ by Wilcox in theoretical organic chemistry. Let $e=u v$ be an edge of a bipartite graph $G$. Gutman proved that $G$ satisfies one of the following relations:

$$
\begin{gathered}
L(G)=L(G-e)+L(G-u-v), \\
L(G)=L(G-e)-L(G-u-v), \\
L(G)=-L(G-e)+L(G-u-v),
\end{gathered}
$$

where $G-e$ (resp. $G-u-v$ ) is the graph obtained from $G$ by deleting edge $e$ (resp. vertices $u$ and $v$ ). In this short note, we obtain a similar result and prove that for any $u_{1}, u_{2} \in U, v_{1}, v_{2} \in V, G$ satisfies one of the following relations:

$$
\begin{gathered}
L(G) L\left(G-u_{1}-u_{2}-v_{1}-v_{2}\right)= \\
L\left(G-u_{1}-v_{1}\right) L\left(G-u_{2}-v_{2}\right)+L\left(G-u_{1}-v_{2}\right) L\left(G-u_{2}-v_{1}\right), \\
L(G) L\left(G-u_{1}-u_{2}-v_{1}-v_{2}\right)= \\
L\left(G-u_{1}-v_{1}\right) L\left(G-u_{2}-v_{2}\right)-L\left(G-u_{1}-v_{2}\right) L\left(G-u_{2}-v_{1}\right), \\
L(G) L\left(G-u_{1}-u_{2}-v_{1}-v_{2}\right)= \\
-L\left(G-u_{1}-v_{1}\right) L\left(G-u_{2}-v_{2}\right)+L\left(G-u_{1}-v_{2}\right) L\left(G-u_{2}-v_{1}\right) .
\end{gathered}
$$
\end{abstract}

\section{Introduction}

Let $G=(U, V)$ be a bipartite graph with bipartition $U \cup V$, if not specified. The algebraic structure count of $G$, denoted by $L(G)$, is defined as the square root of the absolute value of the determinant of the adjacency matrix $A(G)$ of $G[11,12,15,16]$, that is,

$$
L(G)=\sqrt{|\operatorname{det}(A(G))|} .
$$


Let $|U|=m,|V|=n$. It is well known that $L(G)^{2}=(-1)^{n} \operatorname{det}(A(G))$ if $m=n$ and $L(G)=0$ otherwise [8]. Hence we may assume that $G=(U, V)$ is a bipartite graph with $|U|=|V|=n$. If $G$ is a benzenoid graph, then

$$
K(G)^{2}=(-1)^{n} \operatorname{det}(A(G))
$$

where $K(G)$ is the number of perfect matchings (Kekulé structures) of $G$ [5]. Hence if $G$ is a benzenoid graph, then

$$
L(G)=K(G)
$$

The algebraic structure count of a bipartite graph is related closely the thermodynamic stability of the corresponding alternant hydrocarbons. It has important applications in theoretical organic chemistry $[7,10,11,14,17]$. In particular, if $L(G)=0$, then the respective hydrocarbon is extremely reactive and usually does not exist $[10,17]$. See for example some related references $[1-4,9,13]$ on the algebraic structure count.

Note that for any edge $e=u v$ in a graph $G, K(G)$ satisfies the following recurrence relation

$$
K(G)=K(G-e)+K(G-u-v)
$$

where $G-e$ (resp., $G-u-v$ ) is the graph obtained from $G$ by deleting edge $e$ (resp., vertices $u$ and $v$ ). Motivated by this result, Gutman [9] proved that $L(G), L(G-e)$ and $L(G-u-v)$ conform to one of the following three relations:

$$
\begin{gathered}
L(G)=L(G-e)+L(G-u-v), \\
L(G)=L(G-e)-L(G-u-v), \\
L(G)=-L(G-e)+L(G-u-v),
\end{gathered}
$$

In linear algebra, the well-known Dodgson's determinant-evaluation rule [6] implies that, for any square matrix $M=\left(m_{i j}\right)_{n \times n}$ of order $n$,

$$
\operatorname{det}(M) \operatorname{det}\left(M_{1 n}^{1 n}\right)=\operatorname{det}\left(M_{1}^{1}\right) \operatorname{det}\left(M_{n}^{n}\right)-\operatorname{det}\left(M_{1}^{n}\right) \operatorname{det}\left(M_{n}^{1}\right)
$$

where $M_{1 n}^{1 n}$ (resp., $M_{i}^{j}$ ) is the matrix obtained from $M$ be deleting two rows and two columns 1 and $n$ (resp., the $i$ th row and $j$ th column).

Motivated by Eqs. (5)-(8), in this note, we obtain a similar result to Eqs. (5)-(7) and prove that for any $u_{1}, u_{2} \in U, v_{1}, v_{2} \in V$ in a bipartite graph $G=(U, V), G$ satisfies the 
one of the following relations:

$L(G) L\left(G-u_{1}-u_{2}-v_{1}-v_{2}\right)=L\left(G-u_{1}-v_{1}\right) L\left(G-u_{2}-v_{2}\right)+L\left(G-u_{1}-v_{2}\right) L\left(G-u_{2}-v_{1}\right)$,

$L(G) L\left(G-u_{1}-u_{2}-v_{1}-v_{2}\right)=L\left(G-u_{1}-v_{1}\right) L\left(G-u_{2}-v_{2}\right)-L\left(G-u_{1}-v_{2}\right) L\left(G-u_{2}-v_{1}\right)$,

$L(G) L\left(G-u_{1}-u_{2}-v_{1}-v_{2}\right)=-L\left(G-u_{1}-v_{1}\right) L\left(G-u_{2}-v_{2}\right)+L\left(G-u_{1}-v_{2}\right) L\left(G-u_{2}-v_{1}\right)$.

\section{Proof of the main result}

Note that $G=(U, V)$ is a bipartite graph. If $|U| \neq|V|$, then $L(G)=L\left(G-u_{1}-u_{2}-\right.$ $\left.v_{1}-v_{2}\right)=L\left(G-u_{1}-v_{1}\right)=L\left(G-u_{2}-v_{2}\right)=L\left(G-u_{1}-v_{2}\right)=L\left(G-u_{2}-v_{1}\right)=0$. Hence we can assume that $|U|=|V|=n$. Let $U=\left\{u_{1}^{\prime}, u_{2}^{\prime}, \ldots, u_{n}^{\prime}\right\}, V=\left\{v_{1}^{\prime}, v_{2}^{\prime}, \ldots, v_{n}^{\prime}\right\}$. The bipartite adjacency matrix of $G$, denoted $B(G)=\left(b_{i j}\right)_{n \times n}$, is defined as follows:

$$
b_{i j}= \begin{cases}1 & \text { if } u_{i}^{\prime} v_{j}^{\prime} \text { is an edge of } G, \\ 0 & \text { otherwise. }\end{cases}
$$

Obviously, the adjacency matrix of $G$ can be expressed by

$$
A(G)=\left(\begin{array}{cc}
0 & B(G) \\
B(G)^{T} & 0
\end{array}\right),
$$

where $B(G)^{T}$ is the transpose of $B(G)$. Hence

$$
\operatorname{det}(A(G))=(-1)^{n} \operatorname{det}(B(G))^{2}
$$

By Eqs. (1) and (12),

$$
L(G)=|\operatorname{det}(B(G))|
$$

Without loss of generality, set $u_{1}^{\prime}=u_{1}, u_{n}^{\prime}=u_{2}, v_{1}^{\prime}=v_{1}, v_{n}^{\prime}=v_{2}$. Using Dodgson's determinant-evaluation rule Eq. (8) to $B(G)$, then

$$
\operatorname{det}(B(G)) \operatorname{det}\left(B(G)_{1 n}^{1 n}\right)=\operatorname{det}\left(B(G)_{1}^{1}\right) \operatorname{det}\left(B(G)_{n}^{n}\right)-\operatorname{det}\left(B(G)_{1}^{n}\right) \operatorname{det}\left(B(G)_{n}^{1}\right)
$$

where $B(G)_{1 n}^{1 n}$ (resp., $B(G)_{i}^{j}$ ) is the matrix obtained from $B(G)$ be deleting two rows and two columns 1 and $n$ (resp., the $i$ th row and $j$ th column). By the definition of the bipartite adjacency matrix of a bipartite graph, it is not difficult to see that $B(G)_{1 n}^{1 n}$ (resp., 
$\left.B(G)_{1}^{1}, B(G)_{n}^{n}, B(G)_{1}^{n}, B(G)_{n}^{1}\right)$ is the bipartite adjacency matrix of $G-u_{1}-u_{2}-v_{1}-v_{2}$ (resp., $G-u_{1}-v_{1}, G-u_{2}-v_{2}, G-u_{1}-v_{2}, G-u_{2}-v_{1}$ ). Hence, by Eq. (13),

$$
\begin{gathered}
L\left(G-u_{1}-u_{2}-v_{1}-v_{2}\right)=\left|\operatorname{det}\left(B(G)_{1 n}^{1 n}\right)\right|, \\
L\left(G-u_{1}-v_{1}\right)=\left|\operatorname{det}\left(B(G)_{1}^{1}\right)\right|, \\
L\left(G-u_{2}-v_{2}\right)=\left|\operatorname{det}\left(B(G)_{n}^{n}\right)\right|, \\
L\left(G-u_{1}-v_{2}\right)=\left|\operatorname{det}\left(B(G)_{1}^{n}\right)\right|, \\
L\left(G-u_{2}-v_{1}\right)=\left|\operatorname{det}\left(B(G)_{n}^{1}\right)\right|,
\end{gathered}
$$

Note that $L(G) L\left(G-u_{1}-u_{2}-v_{1}-v_{2}\right) \geq 0$. Then Eqs. (9), (10) and (11) are immediate from Eqs. (13)-(19).

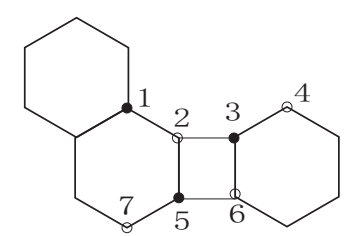

Figure 1. A molecular graph $G$.

\section{Discussion}

For the molecular graph $G$ illustrated in Figure 1, it is not difficult to show that it satisfies: $L(G)=4, L(G-1-2-3-4)=1, L(G-2-3-5-6)=2, L(G-1-2-5-7)=$ $2 ; L(G-1-2)=2, L(G-3-4)=3, L(G-1-4)=1, L(G-2-3)=2, L(G-5-6)=$ $2, L(G-2-5)=4, L(G-3-6)=3, L(G-5-7)=2, L(G-1-7)=1$. Hence

$$
\begin{aligned}
& L(G) L(G-1-2-5-7)=L(G-1-2) L(G-5-7)+L(G-1-7) L(G-2-5), \\
& L(G) L(G-1-2-3-4)=L(G-1-2) L(G-3-4)-L(G-1-4) L(G-2-3), \\
& L(G) L(G-2-3-5-6)=-L(G-2-3) L(G-5-6)+L(G-2-5) L(G-3-6) .
\end{aligned}
$$

Thus, a natural question is: For any $u_{1}, u_{2} \in U, v_{1}, v_{2} \in V$ in a bipartite graph $G=(U, V)$, how to determine which equation among Eqs. (9)-(11) holds?

Acknowledgments: The author was supported in part by NSFC Grant (12071180). We are thankful to the anonymous referee for his (or her) revised suggestions. 


\section{References}

[1] O. Bodroža-Pantić, Algebraic structure count of some cyclic hexagonal-square chains on the Möbius strip, J. Math. Chem. 41 (2007) 283-294.

[2] O. Bodroža-Pantić, S. J. Cyvin, I. Gutman, A formula for the algebraic structure count of multiple phenylenes, MATCH Commun. Math. Comput. Chem. 32 (1995) 47-58.

[3] O. Bodroža-Pantić, I. Gutman, S. J. Cyvin, Algebraic structure count of some nonbenzenoid conjugated polymers, ACH Models Chem. 133 (1996) 27-41.

[4] O. Bodroža-Pantić, A. Ilic-Kovacevic, Algebraic structure count of angular hexagonal-square chains, Fibonacci Quart. 45 (2007) 3-9.

[5] M. J. S. Dewar and H. C. Longuet-Higgins, The correspondence between the resonance and molecular orbital theories, Proc. Roy. Soc. (London) A 214 (1952) $482-$ 493.

[6] C. L. Dodgson, Condensation of determinants, Proc. Roy. Soc. London 15 (1866) $150-155$.

[7] A. Graovac, I. Gutman, N. Trinajstić, Topological Approach to the Chemistry of Conjugated Molecules, Springer-Verlag, Berlin, 1977.

[8] A. Graovac, I. Gutman, The determinant of the adjacency matrix of a molecular graph, MATCH Commun. Math. Comput. Chem. 6 (1979) 49-73.

[9] I. Gutman, Note on algebraic structure count, Z. Naturforsch. 39a (1984) 794-796.

[10] I. Gutman, N. Trinajstić, C. F. Wilcox, Graph theory and molecular orbits, X. The number of Kekulé structures and the thermodynamic stability of conjugated systems, Tetrahedron 31 (1975) 143-146.

[11] W. C. Herndon, Resonance theory and the enumeration of Kekulé structures, J. Chem. Edu. 51 (1974) 10-15.

[12] W. C. Herndon, Enumeration of resonance structures, Tetrahedron 29 (1973) 3-12.

[13] D. J. Klein, T. G. Schmalz, S. EI-Basil, M. Randić, N. Trinajstić, Kekulé count and algebraic structure count for unbranched alternant cata-fusenes, J. Mol. Struct. (Theochem) 179 (1988) 99-107.

[14] C. F. Wilcox, A topological definition of resonance energy, Croat. Chem. Acta 47 (1975) 87-94. 
[15] C. F. Wilcox, Stability of molecules containing (4n)-rings, Tetrahedron Lett. 9 (1968) 795-800.

[16] C. F. Wilcox, Stability of molecules containing nonalternant rings, J. Am. Chem. Soc. 91 (1969) 2732-2736.

[17] C. F. Wilcox, I. Gutman, N. Trinajstić, Graph theory and molecular orbitals, XI. Aromatic substitution, Tetradedron 31 (1975) 147-152. 